\title{
General Psychiatry Discordance between family report and clinical assessment of suicide attempts: a prospective study from the emergency department
}

\author{
Yue Zheng (D) , ${ }^{1,2}$ Haiyin Zhang, ${ }^{2}$ Qing Fan ${ }^{2,3}$
}

To cite: Zheng Y, Zhang H, Fan $\mathrm{Q}$. Discordance between family report and clinical assessment of suicide attempts: a prospective study from the emergency department. General Psychiatry 2021;34:e100576. doi:10.1136/ gpsych-2021-100576

Received 20 May 2021 Accepted 08 0ctober 2021

Check for updates

(c) Author(s) (or their employer(s)) 2021. Re-use permitted under CC BY-NC. No commercial re-use. See rights and permissions. Published by BMJ.

${ }^{1}$ East China University of Science and Technology,

Shanghai, China

${ }^{2}$ Shanghai Mental Health Center, Shanghai Jiao Tong University School of Medicine, Shanghai, China

${ }^{3}$ Shanghai Key Laboratory of Psychotic Disorders, Shanghai, China

Correspondence to

Dr Qing Fan;

fanqing@smhc.org.cn

\section{ABSTRACT}

Background Developing accurate identification methods for individuals with suicide attempts and providing them with follow-up care and supports can be a vital component of all comprehensive suicide prevention strategies. However, because of the difficulties concerning one's intentions behind injurious behaviour, identifying suicide attempts is a challenge for families and clinicians. Aims The aim of this study was to investigate the differences between family report and clinical assessment for suicide attempts in the emergency department (ED). Methods A total of 148 patients with suspected suicide attempts (SSAs) and 148 family caregivers in the ED were enrolled. The suicide risk module of the Chinese version of the MINI International Neuropsychiatric Interview and the self-report measure were used to assess those with SSA's suicidal behaviours. The Family Adaptability and Cohesion Evaluation Scales and semi-structured interviews were used to investigate the characteristics of suicide risk and demographics of patients with SSA, as well as the rate and influencing factors of omitted suicide attempts reported by family caregivers.

Results The underreporting rate for family reported suicide attempts in the ED was $69.0 \%$. The suicide attempts group indicated lower mean scores on perceptions of family resources, adaptability and cohesion. Patients' suicide risk rating ( $\mathrm{OR}=0.152,95 \% \mathrm{Cl}: 0.037$ to $0.620, p=0.009)$, adult-children relationship $(0 R=5.037$, $95 \% \mathrm{Cl}: 1.478$ to $17.167, \mathrm{p}=0.010$ ) and caregiver's age ( $\mathrm{OR}=0.279,95 \% \mathrm{Cl}: 0.103$ to $0.757, \mathrm{p}=0.012$ ) might be associated with underreporting by families. If patients committed suicide attempts through a falling injury or medication overdose, their families may have misreported the suicide attempt.

Conclusions The discordance of suicide attempt records between family report and clinical assessment reveals the limitations of family self-reports when identifying suicide attempts. Interviews and observations, together with information from certain diagnoses, should be combined to accurately identify suicide attempters in the ED.

\section{INTRODUCTION}

Suicide is a significant public health concern worldwide. Research describes that over 800000 people commit suicide every year around the world. There are indications that for each adult who actually commits suicide, there are 20 others who have attempted suicide. ${ }^{1}$ It is a fact that suicide attempts are more frequent and even more underestimated than suicide itself. ${ }^{2}$ Prior history of suicide attempts is the most significant predictor of death by suicide in the general population: individuals who have made prior suicide attempts are at much higher risk of dying by suicide than individuals who have not made prior attempts. ${ }^{1}$ Thus, developing accurate identification methods for these high-risk individuals with suicide attempts and providing them with follow-up care and support can be a vital component of all comprehensive suicide prevention strategies. However, the formal definition of suicide attempt is not straightforward. ${ }^{3}$ Previous research has discussed that non-fatal suicidal thoughts and behaviours could be classified into three categories-suicide ideation: thoughts of engaging in behaviour intending to die; suicide plan: the formulation of a specific method by which one intends to end one's life; and suicide attempt: engagement in potentially self-injurious behaviour through which there is at least some intent to end one's life. ${ }^{3}$ This definition emphasised one's deliberate intention to die by suicide attempt. Because of the difficulties concerning one's intentions behind injurious behaviour, identifying suicide attempts is challenging for both family members and clinicians.

Some sociodemographic characteristics such as gender, age, marital status, family relationship and occupational status may predict one's risk of attempting suicide. ${ }^{1}$ Collection of demographic data, assessment of mental conditions and analysis of methods used for suicide attempts provide essential information in evaluating and preventing the risk of suicidal behaviours. Current research indicates two primary methods for 
collecting information about suicide attempts: from self-reports of suicidal behaviour in surveys of representative samples and from treatment medical records. ${ }^{1}$ Since there are no standard methods for clinically diagnosing and recording suicide attempts, ${ }^{14}$ self-reporting from family caregivers sometimes serves as a relatively convenient tool for collecting emergency data during hospitalisation. In China, demographic data, including causes of harm, are often collected from patients' families when admitted to the emergency department (ED). The effectiveness of information is highly relevant to the accuracy of family report, ${ }^{5}$ especially for some 'accidents' associated with suspected suicidal behaviours. However, self-report biases may be introduced when responding to sensitive questions, such as suicidal behaviours. Although self-reports have limitations in risk evaluation, few studies have considered the accuracy of self-reported assessment in suicide attempts, not to mention the research conducted on evaluating the underreporting of family reports for suicide attempts. A retrospective analysis of the ED registry in Shanghai from 2007 to 2010 reported that only $0.4 \%$ of deliberate self-harm cases had an ED diagnosis of 'attempted suicide'. ${ }^{6}$ A nationwide epidemiological study found that in China, $5.4 \%$ of deaths recorded as specific types of accidents were, in fact, suicides. ${ }^{7}$ These studies reveal the possibilities of underestimated data. However, they have not been conducted comparing the coherence of family self-reports of suicide attempts with clinical assessment in a population-based study.

Patients with suicide attempts are usually sent to the ED for treatment. Prior studies have shown that considerable numbers of patients visited the ED in the year before their suicide due to non-fatal self-harm. However, the episodes may not have resulted in contact with mental health services. ${ }^{8}$ Several effective mental health strategies for suicide prevention have been discussed,${ }^{8-10}$ and there is an increasing recognition of the need for involvement of the entire community. Multiple resources, including community support, such as family and medical centres, should play active roles in suicide prevention. However, research on the role of family caregivers in the treatment of suicidal behaviours and the efficacy of family involvement is scant. ${ }^{11}$ Although some 'Gatekeeper' programmes emphasise the importance of suicidal prevention education for families, ${ }^{12}$ much of the clinical research on families who had a member commit suicide focused on how to help members deal with the emotional burden after suicide. ${ }^{13}$

Families always play a crucial role in caregiving and help-seeking for patients with suicide attempts. ${ }^{58}$ Nevertheless, the fear of unwanted impacts, such as stigma, lack of insurance coverage for suicidal behaviour or concern about potential legal complications, may lead them to hide patients' suicidal behaviours. ${ }^{14}$ Besides, some patients with suicide attempts may conceal their intentions or behaviours over an extended period; as a result, their behaviours may not be alarming for families. ${ }^{15}$ Thus, estimating the underreporting rate in selfreported suicide attempts by family members compared with clinically identifying suicide attempts will help families and clinicians improve the precision of identifying the risk of suicide. Simultaneously, understanding the challenges families face and their resources through analysing their expression and description can be valuable in selecting appropriate psychological assistance.

This study explored the discordance between family report and clinical assessment for suicidal behaviours in the ED. First, we examined whether the family caregivers of patients with suicide attempts had an increased possibility for underreporting suicidal behaviours, compared with family caregivers of those patients who had accidental injuries (to control for the similar suddenness of injuries). Second, we hypothesised that the underreporting rate was related to the suicide attempt (SA) group with particular psychological characteristics related to patients.

\section{METHODS}

\section{Ascertainment}

According to previous evidence, ${ }^{16}$ suspected suicidal behaviours are considered when the following 17 diagnoses are evident: suicide attempts, ingestion of chemicals, ingestion of unknown poison, rodenticide ingestion, pesticide ingestion, ingestion of foreign bodies, drug overdose, medication overdose, attempted/accidental hanging, attempted/accidental drowning, falling injury, food refusal, laceration of the hand, laceration to the neck, alcohol poisoning, carbon monoxide poisoning and other miscellaneous injuries. ${ }^{6}$ In this study, we used this evidence as a grouping criteria to classify all patients as 'SSA (suspected suicide attempts)' or 'non-SSA'. If patients' diagnoses (according to admission records) fit these criteria with informed consent (all the individuals were told the aim of the research), they were included as SSA and became the subjects of investigation. In the present study, all data were collected within 7 days after admission. To improve compliance, the ED nurses were employed as referrers but were not asked to participate in interviews. The data collection was conducted by a team that received assessment training. This team consisted of attending doctors and a core researcher (out-of-hospital staff).

\section{Participants}

We collected the case data from August 2016 to June 2017 in the EDs of three general hospitals in Shanghai, China: Shanghai General Hospital North Campus, Shanghai General Hospital South Campus and Shanghai Sixth People's Hospital. All three hospitals are among the largest general hospitals (more than 1750 beds) in Shanghai, serving a rapidly expanding population.

According to the hospital ED information system, a total of $874 \mathrm{ED}$ patients with one of 17 diagnoses (SSA) 
874 Emergency Department patients with 1 of 17 diagnoses (suspected suicide attempts, SSA) registered from August 2016 to June 2017

726 excluded:

- 203 patients had persistent cognitive

impairment that made it impossible to administer

survey.

- 127 patients had severe physical diseases.

- 396 patients declined to participate in the study.

148 patients (and 148 family caregivers of these patients) participated in the study

-84 cases were classified into the suicide attempts group (SA, 56.8\%).

- 64 cases were classified into the non-suicidal risk group (NR, 43.2\%).

Figure 1 Flowchart of the study.

were registered from 1 August 2016 to 30 June 2017. In this study, 726 patients were excluded: 203 patients had persistent cognitive impairment that made it impossible to administer the survey; 127 patients had severe physical diseases; 396 declined to participate in the study. In total, 296 individuals voluntarily participated in the research with informed consent, including 148 patients $(16.9 \%)$ and 148 family caregivers of these patients. The sample size in this study was determined to be sufficient through calculations by GPower V.3.1 software, ${ }^{17}$ which gave the statistical power of 0.95 . For those less than 18 years of age, informed consent was obtained from their parents (see figure 1).

The inclusion criteria for patients' family caregivers were as follows: (1) essential members of the patient's family (spouse, parents, siblings); (2) principal ED caregivers; (3) informed consent. A descriptive, exploratory and comparative study was conducted in this convenience sample.

\section{Instruments and assessment procedure}

Considering the difficulty of filling out questionnaires for emergency room patients, structured interviews were used to collect information in person. Each interview lasted, on average, $30 \mathrm{~min}$. The suicidality module of the Chinese version of the MINI International Neuropsychiatric Interview (MINI), Version $5.0^{18}{ }^{19}$ was used to assess the suicide risk and its severity within the month preceding the interview. This Chinese version's reliability and validity have been well established, with good test-retest reliability $(0.97-1.00)$ and validity $(0.76-0.88) .{ }^{18}$ MINI suicidality module has six items, and the total suicide risk score is 33 .

In this study, the clinical assessment of suicide attempts among patients with SSA was based on MINI and the experienced attending doctor's evaluation (the reviewing contents: anamnesis, medication and counselling information in clinical records). According to this assessment, the patients with SSA were classified into two groups: SA group (a response of "Yes" to the C5 item in MINI: Did you attempt suicide before hospitalization?) and the non-suicidal risk group (NR) (a response of "No" to the C5 item in MINI).$^{20}$ Following previous studies, ${ }^{21}{ }^{22}$ the SA group could be classified into impulsive (suicide attempters without a history of persistent ideation) and non-impulsive suicide attempts (suicide attempters with a history of suicidal ideation that persisted for a week or more). The non-impulsive acts were carried out with greater intent to risk. ${ }^{22}$ Also, non-impulsive suicide attempts were dichotomised as medium suicidal risk (a response of "No" to the C6 item in MINI) and high suicidal risk classes (a response of "Yes" to the C6 item in MINI), as measured via the history of the previous suicide attempt. In this study, the three subgroups (impulsive risk, medium risk and high risk) were compared with one another and with the non-suicidal risk group (NR).

The Family Adaptability and Cohesion Evaluation Scales (FACES II-CV) was used to measure the family adaptability (14 items) and cohesion (16 items) completed by family caregivers. The higher scores indicate better family adaptability and cohesion. This Chinese version's reliability and validity have been well established, with a good internal consistency (0.73$0.85)$, test-retest reliability (0.84-0.91) and convergent validity $(0.54-0.68){ }^{23}$ In this study, Cronbach's $\alpha$ in this study was: 0.84 (adaptability) and 0.82 (cohesion).

The structured interview for family caregivers was used to evaluate the accuracy, objectivity and transparency of the family reports of suicide attempts in a standardized manner, asking the following questions:

1. Describe the reasons for the patients' hospitalisation (Please distinguish whether it is a suicidal behaviour).

2. Do you mind letting others know that a family member is admitted to the hospital? 
3. Are you thinking about telling your friends and family what is going on?

Additional predictor variables include demographic and clinical factors of patients and their family caregivers. During the investigation, the patients and their family caregivers were interviewed separately. Medical history (including mental illness, family history of suicide, that information conducted by experienced attending doctor's clinical assessment and evaluation referred from anamnesis, medication and counselling information in clinical records) and behavioural information were also collected for patients. All of the options were obtained from the pre-survey interviews.

\section{Data analysis}

IBM SPSS Statistics (V.24) was used for statistical analysis of all data. The age, gender, education background and occupation of the total samples were described and statistically analysed. One-way or two-way ANOVA was used to compare the differences of groups. Chisquared test and Fisher's exact test were used to compare proportions. Standard descriptive statistical methods were used to compare demographic data. Logistic regression models were used to identify variables associated with family report. All $p$ values were two tailed and set at 0.05 for statistical significance.

\section{RESULTS}

Among 148 patients of suspected suicide attempts in the study, 84 cases were assessed to be suicide attempts and classified into the SA (suicide attempts) group $(84,56.8 \%)$ and the NR (non-suicidal risk) group (64, $43.2 \%$ ). Based on SA's responses to MINI, 84 cases in the SA group was divided into three subgroups: 14 cases in the impulsive-risk class, 16 cases in the medium-risk class and 54 cases in the high-risk class.

Table 1 provides sociodemographic information of patients in the SA and NR groups. For patients, ten categories of variables were measured in the questionnaire, which contained gender, age, education level, marital status, occupational status, living with family, family satisfaction, monthly family income, place of origin and religious belief. The age, education level, marital status, occupational status, living status and monthly family income indicated no significant difference. Gender distributions $\left(\chi^{2}=12.84, \mathrm{p}=0.005\right)$, family satisfaction $(\mathrm{p}<0.001)$, place of origin $\left(\chi^{2}=22.60\right.$, $\mathrm{p}<0.001)$ and religious belief $\left(\chi^{2}=8.08, \mathrm{p}=0.044\right)$ variables were significantly different by group. Although there were more males than females in both SA and NR, high-risk classes had the most females $(n=28$, $51.9 \%$ ). For family caregivers in SA, $58.3 \%$ of individuals were female $(n=49), 41.7 \%$ of individuals were male, $39.3 \%$ were younger than 30 years old $(n=33)$, $40.5 \%$ were between 30 and 59 years old $(n=34)$, and $20.2 \%$ were older than 60 years old $(n=17)$.

\section{Clinical assessment of patients}

The patients' clinical assessment of SA and NR based on information from the clinical interview are shown in table 2. These two groups differed for many of the variables. Suicide attempts mainly took place at home $(n=66)$ and demonstrated a higher incidence of medication overdose $(n=26)$, falling injury $(n=35)$ and pesticide ingestion $(n=11)$. Differences in being diagnosed with a mental illness were statistically significant among four groups $(p<0.001)$, and mental illness was also more likely in the high-risk group $(\mathrm{n}=24,44.4 \%)$. In the highrisk group, family conflicts $(\mathrm{n}=25)$ and mental illness $(n=11)$ were the two leading causes of suicide attempts. Other causes were mood swings $(n=5)$, work or study pressure $(n=3)$, interpersonal relationships $(n=1)$ and crisis events $(n=1)$.

\section{Caregivers' reports of suicide behaviours and attitudes}

The comparison between family caregivers' reports of suicide behaviours and attitudes in SA and NR is shown in table 3 . Considering clinical assessment as a standard, the total negative reporting of $69.0 \% \quad(n=58)$ in the SA group $(\mathrm{n}=84)$ meant the underreporting rate of suicide attempts-about $69.0 \%$ of cases with suicide attempts could be undetected if only according to family report (table 3). Particularly, in the impulsive risk group, all the caregivers in this study negatively reported suicide attempts. Families from the SA group would possibly mind letting others know about being admitted to hospital $(n=62)$ and talking about these issues $(\mathrm{n}=60)$ with others, particularly in mediumrisk $(\mathrm{n}=13,81.3 \% ; \mathrm{n}=12,75.0 \%)$ and high-risk groups $(\mathrm{n}=43,79.6 \% ; \mathrm{n}=42,77.8 \%)$. Compared with the NR group, the SA group indicated lower mean scores in family resources (4.92 (2.52)), family adaptability perceptions (33.62 (15.19)) and family cohesion (50.68 (16.42)).

\section{Discordance between family report and clinical assessment}

The discordance between clinical assessment and family report in the SA group was compared as shown in table 4 . When patients were diagnosed with falling injury $(n=32,55.2 \%)$ and medication overdose $(n=15$, $25.9 \%$ ), family reports demonstrated mostly underreporting. In addition, all the participants were required to describe the reasons for which patients were admitted to the emergency department. Interview data collected showed that family caregivers tended to underreport suicidal behaviours $(\mathrm{n}=58)$ and express the reasons as accidental falling $(n=31)$, drug misuse $(n=16)$, unknown cause $(n=5)$, food poisoning $(n=3)$, impulse injury $(n=1)$, alcohol abuse $(n=1)$, or accidental injury $(\mathrm{n}=1)$.

Dependent variables were established to explore the possible factors influencing the underreporting of suicide attempts, including taking the patients' risk of suicide and diagnoses records, demographic data of patients and their primary family caregivers, 
Table 1 Sociodemographic variables: comparison between patients in SA and NR (n (\%))

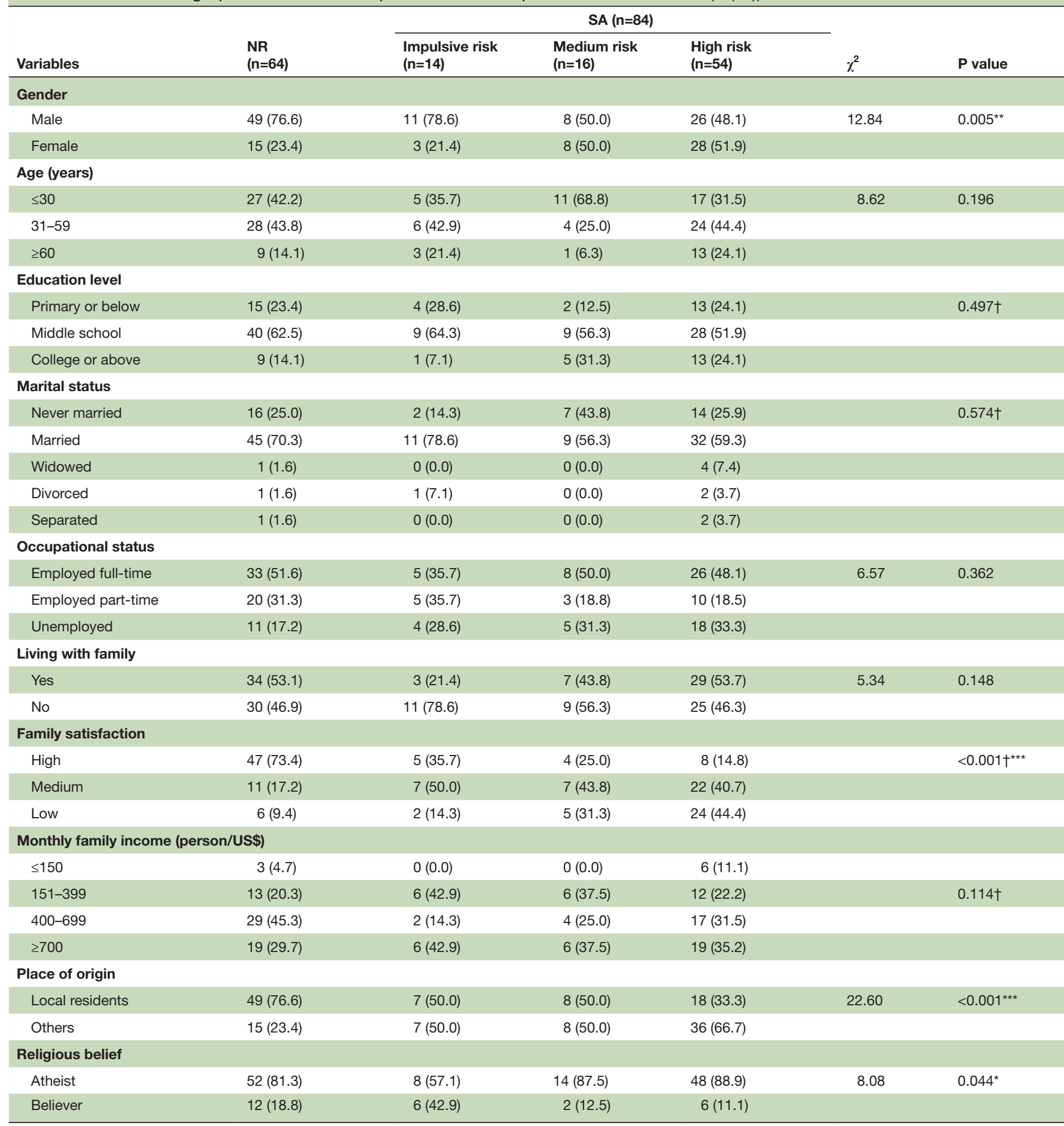

${ }^{*} \mathrm{p}<0.05,{ }^{* *} \mathrm{p}<0.01,{ }^{* * *} \mathrm{p}<0.001$

†Fisher's exact test.

NR, non-suicidal risk; SA, suicide attempts.

and information from family records (relationship to patients, family resources, family cohesion, family adaptability). According to univariate analysis, 17 independent variables indicated significant differences between the two groups (positive SA and negative SA), which were considered the primary influential factors of underreporting. A backward stepwise logistic regression analysis method was then used to select the predicting variables to develop the most appropriate models. Table 5 showed the results of multivariate logistic regression. The Hosmer-Lemeshow test demonstrated good calibration of the logistic regression model $\left(\chi^{2}=5.739, \mathrm{p}=0.571\right)$. In the multivariate analysis, patients' suicide risk rating $(\mathrm{OR}=0.152,95 \%$ CI: 0.037 to $0.620, \mathrm{p}=0.009$ ), adult-children relationship (OR=5.037, 95\% CI: 1.478 to $17.167, \mathrm{p}=0.010)$ 
Table 2 Clinical variables: comparison between patients in SA and NR ( $(\%))$

\begin{tabular}{|c|c|c|c|c|c|}
\hline \multirow[b]{2}{*}{ Variables } & \multirow[b]{2}{*}{ NR $(n=64)$} & \multicolumn{3}{|c|}{ SA $(n=84)$} & \multirow[b]{2}{*}{$P$ value } \\
\hline & & Impulsive risk $(n=14)$ & Medium risk $(n=16)$ & $\begin{array}{l}\text { High } \\
\text { risk }(n=54)\end{array}$ & \\
\hline \multicolumn{6}{|l|}{ Location } \\
\hline Home & $7(10.9)$ & $6(42.9)$ & $14(87.5)$ & $46(85.2)$ & $<0.001 \dagger^{\star \star \star}$ \\
\hline Gathering places & $7(10.9)$ & $4(28.6)$ & $1(6.3)$ & $1(1.9)$ & \\
\hline Outdoor spaces & $21(32.8)$ & $3(21.4)$ & $0(0.0)$ & $2(3.7)$ & \\
\hline Others & 29 (45.3) & $1(7.1)$ & $1(6.3)$ & $5(9.3)$ & \\
\hline \multicolumn{6}{|l|}{ Diagnoses records } \\
\hline Medication overdose & $0(0.0)$ & $0(0.0)$ & $4(25.0)$ & $22(40.7)$ & $<0.001 \dagger^{\star \star \star}$ \\
\hline Pesticide ingestion & $0(0.0)$ & $0(0.0)$ & $2(12.5)$ & $9(16.7)$ & \\
\hline Rodenticide ingestion & $0(0.0)$ & $0(0.0)$ & $0(0.0)$ & $5(9.3)$ & \\
\hline Laceration of the hand & $0(0.0)$ & $0(0.0)$ & $1(6.3)$ & $1(1.9)$ & \\
\hline Falling injury & $16(25.0)$ & $14(100.0)$ & $8(50.0)$ & $13(24.1)$ & \\
\hline Ingestion of other chemicals & $0(0.0)$ & $0(0.0)$ & $0(0.0)$ & $3(5.6)$ & \\
\hline Other miscellaneous injuries & $48(75.0)$ & $0(0.0)$ & $1(6.3)$ & $1(1.9)$ & \\
\hline \multicolumn{6}{|l|}{ Mental illness } \\
\hline No & $63(98.4)$ & $14(100.0)$ & $13(81.3)$ & $30(55.6)$ & $<0.001 \dagger^{\star \star \star *}$ \\
\hline Yes & $1(1.6)$ & $0(0.0)$ & $3(18.8)$ & $24(44.4)$ & \\
\hline \multicolumn{6}{|l|}{ Family history of suicide } \\
\hline Yes & $2(3.1)$ & $0(0.0)$ & $0(0.0)$ & $2(3.7)$ & $1.000 \dagger$ \\
\hline No & $62(96.9)$ & $14(100.0)$ & $16(100.0)$ & $52(96.3)$ & \\
\hline \multicolumn{6}{|l|}{ Reasons $\ddagger$} \\
\hline Family conflicts & $0(0.0)$ & - & $5(41.7)$ & $25(54.3)$ & $<0.001 \dagger^{\star \star *}$ \\
\hline Mood swings & $0(0.0)$ & - & $3(25.0)$ & $5(10.9)$ & \\
\hline Interpersonal relationships & $0(0.0)$ & - & $0(0.0)$ & $1(2.2)$ & \\
\hline Work or study pressure & $0(0.0)$ & - & $3(25.0)$ & $3(6.5)$ & \\
\hline Crisis events & $0(0.0)$ & - & $0(0.0)$ & $1(2.2)$ & \\
\hline Mental illness & $0(0.0)$ & - & $1(8.3)$ & $11(23.9)$ & \\
\hline
\end{tabular}

${ }^{* * *} \mathrm{p}<0.001$.

†Fisher's exact test.

$\ddagger$ Twenty-six cases of suicide attempt patients could not record the reasons.

NR, not-suicidal risk; SA, suicide attempts.

and caregiver's age (OR=0.279, $95 \%$ CI: 0.103 to 0.757 , $\mathrm{p}=0.012$ ) were associated with the family's underreporting for suicide attempts.

\section{DISCUSSION}

\section{Main findings}

Based on the clinical information communicated between families and hospitals, this study reorganised the factors influencing the assessment of suicide attempts. Screening the statistical differences between groups at different levels of risk and the reports of their family members provides a new reference for identifying and preventing suicide attempts in the community.

In this study, female patients made up the majority of the high-risk group. Regarding suicidal behaviour, suicide attempts mainly occurred at home and were associated with medication overdose, falling injury and pesticide ingestion. Some studies in China ${ }^{24}$ also found that the proportion of females in suicide attempts was higher than that of males. Urban residents mainly overdosed on medication as a means of suicide, which is consistent with the results of this study. According to a World Health Organization (WHO) report, the incidence ratio of males' attempting suicide in middle-income countries was $0.3 \%$, and that of females was $0.6 \%$. $^{1}$ This phenomenon is also observed in China. At the same time, female suicide attempters tended to adopt milder methods, such as medication overdose, with higher possibilities of being successfully treated. ${ }^{25}$ This is because medication has a relatively lower case-fatality ratio in China: the vast majority involve ingestion of sleeping medications or antianxiety agents. ${ }^{26}$

Families of those who attempt suicide have a relatively lower function in cohesion and adaptability, which 
Table 3 Reports of suicide behaviours and attitudes: comparison between caregivers in SA and NR ( $n(\%))$

\begin{tabular}{|c|c|c|c|c|c|c|}
\hline & \multicolumn{4}{|c|}{ Clinical assessment } & \multirow[b]{3}{*}{$\mathrm{F} / \chi^{2}$} & \multirow[b]{3}{*}{$P$ value } \\
\hline & \multirow[b]{2}{*}{ NR $(n=64)$} & \multicolumn{3}{|c|}{ SA $(n=84)$} & & \\
\hline & & $\begin{array}{l}\text { Impulsive risk } \\
(n=14)\end{array}$ & $\begin{array}{l}\text { Medium risk } \\
(n=16)\end{array}$ & $\begin{array}{l}\text { High risk } \\
(n=54)\end{array}$ & & \\
\hline \multicolumn{7}{|l|}{ Family report assessment } \\
\hline Positive SA† & $1(1.6)$ & $0(0.0)$ & $2(12.5)$ & $24(44.4)$ & & \\
\hline Negative SA & $63(98.4)$ & $14(100.0)$ & $14(87.5)$ & $30(55.6)$ & & $<0.001 \S^{\star \star \star}$ \\
\hline Adult-children & $27(42.2)$ & $5(35.7)$ & $10(62.5)$ & $23(42.6)$ & 2.742 & 0.433 \\
\hline Other & $37(57.8)$ & 9 (64.3) & $6(37.5)$ & $31(57.4)$ & & \\
\hline \multicolumn{7}{|c|}{ Do you mind letting others know that a family member is admitted to the hospital? } \\
\hline Yes & $11(17.2)$ & $6(42.9)$ & $13(81.3)$ & $43(79.6)$ & 53.04 & $<0.001^{\star \star \star}$ \\
\hline No & $4(6.3)$ & $6(42.9)$ & $12(75.0)$ & $42(77.8)$ & & \\
\hline $\begin{array}{l}\text { Family resources evaluation } \\
(0-10) \text {, mean }(S D)\end{array}$ & $5.70(2.74)$ & & $4.92(2.52)$ & & 3.14 & 0.078 \\
\hline Family cohesion, mean (SD) & $70.98(13.63)$ & & $50.68(16.42)$ & & 52.06 & $<0.001^{\star \star \star}$ \\
\hline Family adaptability, mean (SD) & $52.80(12.78)$ & & 33.62 (15.19) & & 53.57 & $<0.001^{\star \star \star}$ \\
\hline
\end{tabular}

${ }^{* * *} \mathrm{p}<0.001$.

†Positive SA: the family's hospitalisation reports were exactly attempted suicide.

$\ddagger$ Negative SA: the family's hospitalisation reports were discordant with attempted suicide.

§Fisher's exact test.

NR, non-suicidal risk group; SA, suicide attempt group.

indicates that their emotional connection and support system have suffered at different development stages. The ability of the corresponding change was comparatively lower. ${ }^{27}$ Consistent with this result, we found that family conflict is a significant risk for suicide attempts in this study. Simultaneously, a lower level of family satisfaction was reported and there were fewer resources in their families. These results were similar to previous research ${ }^{28} 29$

Table 4 Comparison between family reports towards the given diagnoses in SA ( $n(\%))$

\begin{tabular}{lll}
\hline & \multicolumn{2}{c}{ Family reports in SA } \\
\cline { 2 - 3 } & $\begin{array}{l}\text { Identifying } \\
\text { suicidal } \\
\text { behaviours } \\
\text { (positive SA) } \\
\text { (n=26) }\end{array}$ & $\begin{array}{l}\text { Underreporting } \\
\text { suicidal behaviours } \\
\text { (negative SA) } \\
\text { (n=58) }\end{array}$ \\
\hline Diagnoses recorded & $11(42.3)$ & $15(25.9)$ \\
\hline Pesticide ingestion & $6(23.1)$ & $5(8.6)$ \\
\hline Rodenticide ingestion & $2(7.7)$ & $3(5.2)$ \\
\hline Laceration of the hand & $1(3.8)$ & $1(1.7)$ \\
\hline Falling injury & $3(11.5)$ & $32(55.2)$ \\
\hline $\begin{array}{l}\text { Ingestion of other } \\
\text { chemicals }\end{array}$ & $2(7.7)$ & $1(1.7)$ \\
\hline $\begin{array}{l}\text { Other miscellaneous } \\
\text { injuries }\end{array}$ & $1(3.8)$ & $1(1.7)$ \\
\hline
\end{tabular}

SA, suicide attempt group. which found that family relationships could contribute to suicidal thoughts. Family instability may increase the risk of suicide ${ }^{30}$ because for some people wanting to escape from a family in conflict, suicide could be the only option for them. When family caregivers take care of suicide attempts, a problematic relationship may also lead them to a tough dilemma where work and family cannot be balanced. ${ }^{31}$ Therefore, improving familys' interpersonal relationship and conflict management skills seem imperative. Compared with the NR group, the family caregivers of SA care more about their family issues becoming known and feel it is more difficult to talk about those issues. Affected by cultural taboos, 'suicide' may be regarded as 'disgraceful' by the family. Even if they face difficulties, they will rarely ask others for help. ${ }^{32-34}$ Healthcare staff should pay more attention to the emotion and moods of families, given that most of them would not share their feelings with others. Understanding their motivations may help to build cooperative trust with them.

In this study, mental illness is the second risk factor for suicide attempts. Previous research had similar results that suffering from a mental illness, such as schizophrenia and anxiety/depressive disorders, increases the risk of suicide attempts ${ }^{35} 36$ because those with mental illness may have greater impulsivity and aggressive behaviours. ${ }^{37}$

Recent research has indicated that about $69.0 \%$ of individuals with suicide attempts could be undetected if going strictly by family reports. The underreporting rate 
Table 5 Logistic regression analysis to determine factors associated with the family's underreporting for suicide attempts $(\mathrm{n}=84)$

\begin{tabular}{lllllll}
\hline Factors & B & SE & Wald $\chi^{2}$ & P value & OR & 95\% Cl \\
\hline Patients' suicide risk rating & -1.887 & 0.719 & 6.889 & $0.009^{* *}$ & 0.152 & 0.037 to 0.620 \\
\hline Adult-children relationship & 1.617 & 0.626 & 6.681 & $0.010^{*}$ & 5.037 & 1.478 to 17.167 \\
Caregiver's age & -1.278 & 0.510 & 6.276 & $0.012^{*}$ & 0.279 & 0.103 to 0.757 \\
\hline
\end{tabular}

${ }^{*} \mathrm{p}<0.05,{ }^{* *} \mathrm{p}<0.01$.

OR, odds ratio.

of suicide attempts by families reveals that the family may avoid, conceal or neglect the patients' suicidal behaviours, especially when diagnosed as an accidental falling injury or medication overdose. Expressions commonly occurring in family reports include 'medicine misuse', 'accidental falling down', 'unknown reasons' and 'food poisoning'. Those seem to emphasise the non-intentionality on the part of the victim. This study showed that a lower level of suicide risk, adult-children relationship, and younger caregivers might predict false-negative reporting for SA. Previous research indicated that stigma or other sensitive reasons (to hide potential conflicts) may explain why some family caregivers conceal suicide intentionally. ${ }^{31}{ }^{38}$ Adultchildren relationship indicated that caregivers often played the role of guardians of children and adolescents. The attempted suicide of children or adolescents makes parents feel stressed, guilty and angry, especially when it happens because of a family conflict. ${ }^{39}$ At the same time, younger caregivers often feel more anxious about the future development of those patients influenced by suicide attempt stigma. ${ }^{34}$ These emotions can explain why younger caregivers often underreport patients' suicidal behaviours to reduce self-blame and fears about patients' potential risk. From the public health perspective, recording suicide attempts as 'accidents' might be partly explained by several undesirable factors, such as stigma, lack of insurance coverage and potential legal complications. ${ }^{1}$ Therefore, it was necessary to measure these data for this study. During the survey, the researcher used the Experience of Shame Scale to measure the sense of shame. ${ }^{41}$ However, $89(60 \%)$ families did not complete this scale, and about 50 (34\%) families did not answer the insurance questions. It may indicate these families' avoidance of unwanted consequences. In future research, we will explore training programmes to eliminate the stigma of reporting for families with suicide attempts.

In addition, those low-risk SAs, such as impulsive suicide attempts, cannot be easily detected by families because individuals do not have apparent suicide plans. Lack of communication, stigma and negative coping may also cause families to ignore patients' hidden suicidal intentions. Thus, individual psychotherapy for only suicide attempts is not enough to reduce suicide. A prevention programme should involve families, provide more educational resources on suicide identification and communication management to help them respond to psychological problems, and provide appropriate services in daily life. ${ }^{34}$

\section{Limitations}

Despite the significant contributions of this study, there are limitations. First, because of the sensitive topic of the research, some families were not willing to participate in the study. The lack of a random sample means that the generalisability of these results to other hospitals in China is unclear. Moreover, diverse populations, like minority populations or lesbian, gay, bisexual, transgender and questioning (LGBTQ) individuals, may experience different types of pressure from their families. Second, this study aimed to include all suspected attempted suicides recorded at emergency departments. However, it is difficult to include all eligible patients due to information delay, the patient's inability to answer questions, refusal to participate in the study and other factors. One hundred and forty-eight patients is a relatively small sample size. Not examining prior mental diagnoses of patients might have impacted the analysis of SA motivations. In future research, we will expand the sample size, consider the concrete mental diagnoses, and make an in-depth discussion on how to provide timely and effective interventions for the SA families.

\section{Implications}

This study has clinical implications for working with families. Patients at risk for suicide attempts may not wish to have these facts disclosed by their families. This finding will help clinicians direct multiple measures. Family report should never be used alone, especially those containing accidental falling injury or medication overdose diagnoses. Interviews and observations, together with information from other mental diagnoses, should be combined to evaluate.

It is still a worldwide challenge to screen and assess the risk of suicide through a clinical gold standard. ${ }^{4}$ If healthcare records just document suicide attempts in a binary yes/no interview with the family, there will be important indicators missed. In the high-risk group, suicide attempt interviews showed that most participants suffered from family conflicts, mental illnesses, mood swings, work or study pressure, interpersonal relationships and crisis problems. These findings suggest potential directions for assessment. The ED team should be aware that some families may express suicide attempts differently or deny them. However, they may feel more comfortable disclosing suicide behaviours when approached privately in a supportive, caring manner. ${ }^{42}$ Thus, how clinicians 
respond and treat patients or families when they disclose their suicide attempts is vitally important. The literature suggests that some promising tools are being examined for screening and assessing suicide risk, which would likely be an improvement for families identifying and reporting suicide risk. For example, the magnitude of suicide risk and self-harm fluctuations was studied through an ecological momentary assessment. ${ }^{43}$ For the ED team, more training on evidence-based clinical interviews (suicidal ideation, plan, access to means, intent, lethality, protective factors, previous attempts or aborted/ interrupted attempts and present/past risk factors) ${ }^{44} 45$ and the use of proper objective instruments (eg, Nurses Global Assessment of Suicide Risk) ${ }^{4647}$ are recommended by researchers and professions to increase clinicians' selfconfidence in assessing and screening suicide risk. ${ }^{44}$

Furthermore, this study primarily investigates the epidemiological characteristics and discordance of SA and their family reports. A lower level of suicide risk, younger caregivers, and adult-children relationship may predict underreporting for suicide attempts. These findings highlight the potential limitations of identifying suicide attempts in ED and promoting more specific assistance for SA patients and their families. Few studies have investigated these patterns using reports from SA patients and their families. However, this study observes and highlights such discordance. Further actions can be taken in response to individuals' concrete psychological status, such as psychological assessment, treatment or referral with more caring language. Considering the stigma surrounding suicide, hospitals can offer family self-help material, specialist hospital information, and psychological hotlines.

Acknowledgements We thank Dr Xu Yeqing from Shanghai Mental Health Centre, Dr Feng Qiming from Shanghai Sixth People's Hospital, and our colleagues from Shanghai Mental Health Centres, Shanghai General Hospital North Campus, Shanghai General Hospital South Campus, Shanghai Sixth People's Hospital, who provided insight and expertise that greatly assisted the research.

Contributors All authors have contributed significantly, and all authors agree with the content of the manuscript. YZ developed the concept, designed the study, acquired the data and performed analysis. $\mathrm{HZ}$ obtained the research funding and provided supervision for the study. QF provided statistical advice and editing supports. YZ drafted the manuscript and all authors contributed substantially to its revision. $\mathrm{YZ}$ is the guarantor.

Funding This research was supported by Funding: Three-Year Action Plan for Public Health Project in Shanghai (GWIV-5).

Disclaimer The views and opinions expressed in this report are those of the authors and should not be construed to represent the views of any of the sponsoring organisations, agencies or the government.

Competing interests None declared.

Patient consent for publication Obtained.

Provenance and peer review Not commissioned; externally peer reviewed.

Data availability statement Data are available on reasonable request. Data are available on reasonable request. Please contact corresponding author if data are required.

Open access This is an open access article distributed in accordance with the Creative Commons Attribution Non Commercial (CC BY-NC 4.0) license, which permits others to distribute, remix, adapt, build upon this work non-commercially, and license their derivative works on different terms, provided the original work is properly cited, appropriate credit is given, any changes made indicated, and the use is non-commercial. See: http://creativecommons.org/licenses/by-nc/4.0/.

ORCID iD

Yue Zheng http://orcid.org/0000-0001-7454-1430

\section{REFERENCES}

1 World Health Organization. WHO's preventing suicide: a global imperative report. World Health Organization, 2014.

2 Bertolote JM, Fleischmann A, De Leo D, et al. Repetition of suicide attempts: data from emergency care settings in five culturally different low- and middle-income countries participating in the who SUPRE-MISS study. Crisis 2010;31:194-201.

3 Nock MK, Borges G, Bromet EJ, et al. Suicide and suicidal behavior. Epidemiol Rev 2008;30:133-54.

4 Bernert RA, Hom MA, Roberts LW. A review of multidisciplinary clinical practice guidelines in suicide prevention: toward an emerging standard in suicide risk assessment and management, training and practice. Acad Psychiatry 2014;38:585-92.

5 Le Moal V, Lemey C, Walter M, et al. Viewpoint: toward involvement of caregivers in suicide prevention strategies; ethical issues and perspectives. Front Psychol 2018;9:2457.

$6 \mathrm{Xu}$ Y, Phillips MR, Wang L, et al. Retrospective identification of episodes of deliberate self-harm from emergency room registers in general hospitals: an example from Shanghai. Arch Suicide Res 2013;17:345-59.

7 Wang L-J, Phillips M, Huang Z-J, et al. [Evaluation on the accuracy of reported suicides in the Chinese population]. Zhonghua Liu Xing Bing Xue Za Zhi 2003;24:889-92.

8 Liu J. Need to establish a new adolescent suicide prevention programme in South Korea. Gen Psychiatr 2020;33:e100200.

9 Takahashi Y, Takahashi S, Imamura Y, et al. [The national strategies for suicide prevention by the United Nations/World Health Organization and the present situation of suicide in the East Asia]. Seishin Shinkeigaku Zasshi 2014;116:690-6.

10 Ishimo M-C, Sampasa-Kanyinga H, Olibris B, et al. Universal interventions for suicide prevention in high-income organisation for economic co-operation and development (OECD) member countries: a systematic review. Inj Prev 2021;27:184-93.

11 Frey LM, Cerel J. Risk for suicide and the role of family: a narrative review. J Fam Issues 2013;36:716-36.

12 Rae Swanke J, Melinda Dobie Buila S. Gatekeeper training for caregivers and professionals: a variation on suicide prevention. Adv Ment Health 2010;9:98-104.

13 Buus N, Caspersen J, Hansen R, et al. Experiences of parents whose sons or daughters have (had) attempted suicide. J Adv Nurs 2014;70:823-32.

14 Garcia-Williams AG, McGee RE. Responding to a suicidal friend or family member: a qualitative study of college students. Death Stud 2016;40:80-7.

15 Goldston DB, Molock SD, Whitbeck LB, et al. Cultural considerations in adolescent suicide prevention and psychosocial treatment. Am Psychol 2008;63:14-31.

16 Gray D, Coon H, McGlade E, et al. Comparative analysis of suicide, accidental, and undetermined cause of death classification. Suicide Life Threat Behav 2014;44:304-16.

17 Faul F, Erdfelder E, Lang A-G, et al. G*Power 3: a flexible statistical power analysis program for the social, behavioral, and biomedical sciences. Behav Res Methods 2007;39:175-91.

$18 \mathrm{Si}$ T, Shu L, Dang Y. Evaluation of the reliability and validity of Chinese version of the mini-international neuropsychiatric interview in patients with mental disorders. Chinese Ment Health Journal 2009;23.

19 Sheehan DV, Lecrubier Y, Sheehan KH. The Mini-International neuropsychiatric interview (M.I.N.I.): the development and validation of a structured diagnostic psychiatric interview for DSM-IV and ICD10. J Clin Psychiatry 1998;59 Suppl 20:22-33;quiz 34-57.

20 Ding S, Li X, Hua Y, et al. Risk factors for suicidal tendency in adult patients with epilepsy in China. Epilepsy Behav 2019;97:118-22.

21 Pompili M, Rihmer Z, Akiskal H, et al. Temperaments mediate suicide risk and psychopathology among patients with bipolar disorders. Compr Psychiatry 2012;53:280-5.

22 Wojnar M, llgen MA, Czyz E, et al. Impulsive and non-impulsive suicide attempts in patients treated for alcohol dependence. J Affect Disord 2009;115:131-9.

23 Phillips MR, West CL, Shen Q, et al. Comparison of schizophrenic patients' families and normal families in China, using Chinese 
versions of FACES-II and the family environment scales. Fam Process 1998;37:95-106.

24 Sun L, Zhang J. Gender differences among medically serious suicide attempters aged $15-54$ years in rural China. Psychiatry Res 2017;252:57-62.

25 Zhang J, Jia S, Jiang C, et al. Characteristics of Chinese suicide attempters: an emergency room study. Death Stud 2006;30:259-68.

26 Xue D, Ji H, Fei L. Characteristics of suicide attempts treated over a 7 year period in the emergency department of a general hHospital in Beijing. Sichuan Ment Health 2004;17:95-8.

27 Deng Y, Yang L, Yin Y. Family adaptability and cohesion among rural attempted suicides. Chinese Journal of Public Health 2011;27:184-5.

28 Nagamitsu S, Mimaki M, Koyanagi K, et al. Prevalence and associated factors of suicidality in Japanese adolescents: results from a population-based questionnaire survey. BMC Pediatr 2020;20:467.

29 Lipschitz JM, Yen S, Weinstock LM, et al. Adolescent and caregiver perception of family functioning: relation to suicide ideation and attempts. Psychiatry Res 2012;200:400-3.

30 Mathew A, Saradamma R, Krishnapillai V, et al. Exploring the family factors associated with suicide attempts among adolescents and young adults: a qualitative study. Indian J Psychol Med 2021;43:113-8.

31 Castelli Dransart DA, Guerry S. Help-seeking in suicidal situations: paramount and yet challenging. interactions between significant others of suicidal persons and health care providers. J Clin Med 2017;6. doi:10.3390/jcm6020017. [Epub ahead of print: 13 Feb 2017].

32 Tzeng W-C, Lipson JG. The cultural context of suicide stigma in Taiwan. Qual Health Res 2004;14:345-58.

33 Han J, Batterham PJ, Calear AL, et al. Factors influencing professional help-seeking for suicidality. Crisis 2018;39:175-96.

34 Asare-Doku W, Osafo J, Akotia CS. The experiences of attempt survivor families and how they cope after a suicide attempt in Ghana: a qualitative study. BMC Psychiatry 2017;17:178.

35 Song Y, Rhee SJ, Lee H, et al. Comparison of suicide risk by mental illness: a retrospective review of 14 -year electronic medical records. $J$ Korean Med Sci 2020;35:e402.

36 Yates K, Lång U, Cederlöf M, et al. Association of psychotic experiences with subsequent risk of suicidal ideation, suicide attempts, and suicide deaths: a systematic review and metaanalysis of longitudinal population studies. JAMA Psychiatry 2019;76:180-9.

37 Dávila Cervantes CA, Luna Contreras M. Suicide attempt in teenagers: associated factors. Rev Chil Pediatr 2019;90:606-16.

38 Rober P, Walravens G, Versteynen L. "In search of a tale they can live with": about loss, family secrets, and selective disclosure. J Marital Fam Ther 2012;38:529-41.

39 Greene-Palmer FN, Wagner BM, Neely LL, et al. How parental reactions change in response to adolescent suicide attempt. Arch Suicide Res 2015;19:414-21.

40 Frey LM, Hans JD, Cerel J. An interpretive phenomenological inquiry of family and friend reactions to suicide disclosure. J Marital Fam Ther 2017;43:159-72.

41 Han J, Batterham PJ, Calear AL, et al. Translation and validation of the Chinese versions of the suicidal ideation attributes scale, stigma of suicide scale, and literacy of suicide scale. Death Stud 2017;41:173-9.

42 Heyland M, Delaney KR, Shattell M. Steps to achieve universal suicide screening in emergency departments: a call to action. $J$ Psychosoc Nurs Ment Health Serv 2018;56:21-6.

43 Gee BL, Han J, Benassi H, et al. Suicidal thoughts, suicidal behaviours and self-harm in daily life: a systematic review of ecological momentary assessment studies. Digit Health 2020;6:2055 207620963958:205520762096395.

44 Schatten HT, Gaudiano BA, Primack JM, et al. Monitoring, assessing, and responding to suicide risk in clinical research. J Abnorm Psychol 2020;129:64-9.

45 Santel M, Beblo T, Neuner F, et al. Collaborative assessment and management of suicidality (CAMS) compared to enhanced treatment as usual (E-TAU) for suicidal patients in an inpatient setting: study protocol for a randomized controlled trial. BMC Psychiatry 2020;20:183.

46 van Veen M, van Weeghel I, Koekkoek B, et al. Structured assessment of suicide risk in a psychiatric emergency service: psychometric evaluation of the nurses' global assessment of suicide risk scale (NGASR). Int J Soc Psychiatry 2015;61:287-96.

47 Cutcliffe JR, Barker P. The nurses' global assessment of suicide risk (NGASR): developing a tool for clinical practice. J Psychiatr Ment Health Nurs 2004;11:393-400.

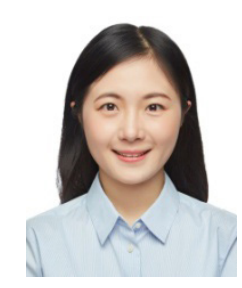

Yue Zheng obtained a master's degree in Psychology from Shanghai Jiao Tong University, Shanghai, China, in 2018. At present, she is a lecturer at East China University of Science and Technology, Shanghai, China. Her main research interests include suicide prevention, mental health, peer support, and mindfulness. 
Correction: Discordance between family report and clinical assessment of suicide attempts: a prospective study from the emergency department

Zheng Y, Zhang H, Fan Q. Discordance between family report and clinical assessment of suicide attempts: a prospective study from the emergency department. General Psychiatry 2021;34:e100576. doi:10.1136/gpsych-2021-100576

This article was previously published with an error. Figure 2 has been replaced with table 5 in the article.

\section{() \\ OPEN ACCESS}

Open access This is an open access article distributed in accordance with the Creative Commons Attribution Non Commercial (CC BY-NC 4.0) license, which permits others to distribute, remix, adapt, build upon this work non-commercially, and license their derivative works on different terms, provided the original work is properly cited, appropriate credit is given, any changes made indicated, and the use is non-commercial. See: http://creativecommons.org/licenses/by-nc/4.0/.

(C) Author(s) (or their employer(s)) 2022. Re-use permitted under CC BY-NC. No commercial re-use. See rights and permissions. Published by BMJ.

General Psychiatry 2022;35:e100576corr1. doi:10.1136/gpsych-2021-100576corr1

A Check for updates 